Post-Print von Doris Pichler (2016): »)Ausweg Fiktion<: Romanzo criminale als Popularisierung eines juristischen Falles«. In: Franziska Stürmer/Patrick Meier (Hg.).: Recht populär. Populärkulturelle Rechtsdarstellungen in aktuellen Texten und Medien.

Baden-Baden: Nomos. 81-104.

\title{
»Ausweg Fiktion«: Romanzo criminale als Popularisierung eines juristischen Falles
}

von

\author{
Doris Pichler
}

\section{Vorbemerkungen}

Seit dem Erscheinen von Romanzo criminale 2002 - der Fiktionalisierung eines der prägendsten Verbrechenskapitel der jüngeren italienischen Geschichte und dessen komplexer juristischer Aufarbeitung, nämlich der Geschichte der berühmt-berüchtigten Banda della Magliana - ist der römische Richter und (nun auch) Schriftsteller Giancarlo De Cataldo aus der Reihe der zeitgenössischen italienischen Bestsellerautoren nicht mehr wegzudenken. In seiner »Berufsautobiographie « In giustizia »gesteht« er, dass sein ursprünglicher Berufswunsch jener des Künstlers gewesen wäre, er sich aber aus rein rationalen Überlegungen für das Jura-Studium entschieden habe:

Warum beschließt ein junger Mann, der eigentlich davon geträumt hatte, Orson Welles [...] zu werden, warum beschließt dieser Mann eines schönen Tages, sich zu einem »Diener des Staates« zu machen? [...] 27 Jahre lang hatte ich es mir in Wahrheit gut gehen lassen: früher oder später musste man das Leben ja ernsthaft angehen. ${ }^{1}$

Diese sprichwörtlichen »zwei Seelen in seiner Brust« ist es ihm mittlerweile zu versöhnen gelungen: Neben seiner Tätigkeit als Richter am Corte d'Assise (dem Schwurgericht) in Rom, ist er als Autor von Kriminalromanen und Thrillern, aber auch von Drehbüchern zu Filmen

1 De Cataldo, In giustizia, 2011, 13, 15. Übersetzungen, sofern nicht anders angegeben, sind meine eigenen. Orig.: »Perché un ragazzo che sognava di diventare Orson Welles [...] un bel giorno pensa di farsi >servitore dello stato<? [...] Per ventisei anni, in fondo ho giocato alla vita: bisognava, prima o poi, fare sul serio $\ll$ 
und Serien über (meist) reale Rechtsfälle der jüngeren italienischen Geschichte eine in den italienischen Medien vielgesehene Persönlichkeit.

Gemeinsam mit illustren Kollegen wie Gianrico Carofiglio, Massimo Carlotto und Carlo Lucarelli, die sich ebenfalls dem Krimigenre verschrieben haben und auch zum Teil - jeder auf seine Art (als Opfer/Täter bzw. als Anwalt oder Richter) - zwischen dem praktizierenden Recht und dessen literarischer Darstellung stehen, wird er nicht müde, die »Ernsthaftigkeit « dieses Genres zu betonen, ${ }^{2}$ denn es ist gerade der Krimi, der die konkrete italienische Verbrechensrealität (und die damit verbundenen sozialen und politischen Themen) zur Diskussion stellen kann. Besonders interessant sind die Verbrechen im Zusammenhang mit den sogenannten Anni di piombo (d.h. der Jahre des linken und rechten Terrorismus in den 1970er und 1980er Jahren und der fragwürdigen Rolle der Geheimdienste und der Freimaurerloge $P 2$ dabei); das Aufdecken der großen Korruptionsfällen in den 1990ern (Stichwort: Tangentopoli und mani pulite) und das daraus resultierende Ende der 1. Republik. Texte De Cataldos, die sich mit solchen Inhalten beschäftigen, wären z.B. die beiden Romane Romanzo criminale (2002) und Nelle mani giuste (2007) sowie die Drehbücher für die Fernsehserien Paolo Borsellino (2004) (um den Mord am Anti-Mafia Richter Paolo Borsellino) und Enzo Tortora. Dove eravamo rimasti (2012) (um einen der spektakulärsten Justizirrtümer der jüngeren italienischen Geschichte).

De Cataldo kann damit als einer der bekanntesten Vertreter einer sogenannten »faktual basierten Verbrechensliteratur ${ }^{3}$ gesehen werden und seine Texte sind Teil einer, um die Worte Elisabeth Tillers zu verwenden, »faktualen Offensive des Fiktionalen«. Mit ihrem Begriff der »faktualen Fiktionen ${ }^{4}$ bezieht sie sich genau auf jene Texte, die nicht zur Gänze aufgearbeitete Fälle der jüngeren italienischen Geschichte zum Thema haben. So sind auch De Cataldos Romane wie auch Film- und Seriendrehbücher von einem politischen und sozialen Engagement geprägt und haben weniger einen einzigen Verbrecher und dessen Überführung zum Inhalt, als vielmehr die »Arbeit« eines ausgeklügelten kriminellen Systems, das bis in höchste Justiz-, Polizei- und Politikerkreise hineinreicht. Sie sind demnach ein ambigues Konstrukt zwischen Real-

2 Vgl. z.B. das Interview mit De Cataldo: »Giancarlo De Cataldo risponde alle domande del Camilleri Fans Club «, URL: http://www.vigata.org/altri_autori/cfc_decataldo.shtml [10.02.2015].

3 Tiller, »Krise und Erneuerung. Die faktuale Offensive des Fiktionalen«, in: lettere aperte 1/2014, 33 (44).

4 Vgl. den Titelzusatz von Tiller, (Anm. 3), 48. 
und fiktiver Geschichte bzw. zwischen geschichtlichen Tatsachen und im Bereich des Fiktionalen wiederzählten geschichtlichen Tatsachen.

»Faktuale Fiktion « beschreibt nur scheinbar einen Widerspruch; vielmehr wird deutlich, dass eine reine Dichotomie Fakt-Fiktion nicht ausreicht, um Fiktionen, wie sie die genannten Autoren liefern, zu erklären. Der Begriff »faktuale Fiktion « weist also auf einen pragmatisch gedachten Fiktionsbegriff hin: Demnach soll Fiktion weder ausschließlich nach textimmanenten Merkmalen, wie z.B. dem der Verwendung des epischen Präteritums, der erlebten Rede u.a., ${ }^{5}$ noch nach semantischen Kriterien (wie dem der Fiktivität) ${ }^{6}$ und damit in Zusammenhang auch nicht ausschließlich nach ontologischen Gesichtspunkten, wie dem der Referenz, ${ }^{7}$ bestimmt werden. Fiktion muss vielmehr pragmatisch gedacht werden, denn sie ist abhängig von der jeweiligen Handhabung, die sie erfährt. ${ }^{8}$ Ein fiktionaler Text schlägt also eine gewisse Rezeptionshaltung vor, die jener beim Spiel ähnlich ist: Beim Spielen wie auch beim Lesen eines fiktionalen Textes ist man sich der Differenz Spiel-Nicht-Spiel jederzeit bewusst, setzt dieses Wissen aber für die Dauer des Spieles außer Kraft; man lässt sich auf ein, im Sinne Kendal Waltons, Spiel des makebelieve ein. ${ }^{9}$ Gibt es genügend Auslöser (meist paratextueller Natur) für solch eine Rezeptionshaltung, tut die Faktenbasiertheit dem ästhetischen Genuss einer fiktionalen Lektüre keinen Abbruch.

Mit seinem Kriminalroman Romanzo criminale nimmt sich De Cataldo nun einer konkreten Verbrechenssituation an, nämlich dem Phänomen der Banda della Magliana - eine ursprünglich aus Kleinkriminellen bestehende unorganisierte Gruppe, die sich zu einer der mächtigsten und brutalsten Verbrecherbanden Roms entwickelte und in den späten 1970er und 1980er Jahren die organisierte Kriminalität Roms unter ihre Kontrolle brachte: Sie kontrollierten das illegale Glücksspiel, die Prostitution und den Drogenhandel und kollaborierten mit der Mafia, der Camorra, aber

5 Vgl. Hamburger, Die Logik der Dichtung, 2. stark veränderte Aufl., 1968, 59, 74.

6 Vgl. Gabriel, Fiktion und Wahrheit. Eine semantische Theorie der Literatur, 1975.

7 Vgl. zur Selbstreferentialität: Genette, Fiktion und Diktion, 1992, 36ff.; Cohn, The Distinction of Fiction, 1999, 13f. Vgl. zum »Rekursargument«: Welsch, »)Wirklich $\measuredangle$. Bedeutungsvarianten - Modelle - Wirklichkeit und Virtualität«, in: Krämer (Hg.), Medien. Computer. Realität, 2. Aufl., 2000, 169.

8 Vgl. Pavel, »Comment définir la fiction«, in: Gefen et al. (Hg.), Frontières de la fiction, 2001, 3 (5): »[...] la fictionnalité dépendrait donc de l'usage qu'on en fait.« Dt.: » [...] die Fiktionalität hängt daher davon ab, wie man sie verwendet«.

9 Vgl. Walton, Mimesis as Make-Believe. On the Foundations of the Representational Arts, 1990. 
auch mit dem italienischen Geheimdienst und der Freimaurerloge $P 2$. Damit ist das Phänomen Banda della Magliana gerade auch auf innenpolitischer Ebene von höchster Brisanz. Ihr »Erfolg« war aber nicht nur den geschickten Verhandlungen mit anderen Verbrecherorganisationen und neuralgischen staatlichen Machtinstanzen zu »verdanken«, sondern auch ihrer privatwirtschaftlichen Organisationsstruktur. Als eine Art »holding criminale« teilte sie einen Teil des Gewinns direkt unter den Mitgliedern auf und »investierte « den Rest wieder in neue »Projekte « (wie z.B. in den Kauf von Drogen, den Aufbau illegaler Casinos etc.).

De Cataldo selbst kam in seiner Funktion als Jurist mit der Banda in Kontakt, als er im Oktober 1995 beim ersten und einzigen maxiprocesso, bei dem 98 Personen auf der Anklagebank saßen, als beisitzender Richter am Schwurgericht tätig war. In seiner bereits erwähnten Berufsautobiographie In giustizia äußert er sich zu diesem Fall folgendermaßen:

»Lieber De Cataldo, in Kürze beginnt der Prozess gegen die Banda della Magliana im Schwurgericht. Wenn Sie Lust haben, dann könnten Sie ihn machen «. Es ist der 20. September 1995. Ich bin 39 Jahre alt. Ich sage ohne zu zögern zu. Zwei Wochen später [...] beginnt der Prozess gegen die mächtigste und berüchtigtste kriminelle Organisation Roms. ${ }^{10}$

Für De Cataldo ist es einer seiner wichtigsten Fälle, der ihn v.a. in seinen schriftstellerischen Ambitionen vorantreibt. In einem Interview mit der deutschen Zeitung Die Zeit sagt er: »Es war eine großartige Chance für mich «, und fügt hinzu: »- als Autor «. ${ }^{11}$ Die »Figuren «- und man beachte die ästhetische Distanz, mit der ein Richter hier von seinen Angeklagten spricht - haben auf ihn von jeher eine Faszination ausgeübt, wie er in einem weiteren Interview betont, und die Bande ist schon während ihres Bestehens, und mehr noch danach, zu einer Legende geworden innerhalb und außerhalb des kriminellen Milieus:

10 De Cataldo, (Anm. 1), 67. Orig.: »)Caro De Cataldo, sta per iniziare in Assise il processo alla Banda della Magliana. Se se la sente potrebbe farlo lei.く È il 20 settembre 1995. Ho trentanove anni. Accetto senza esitare. Quindici giorni dopo [...] si apre il processo alla più potente e famigerata organizzazione criminale romana.

11 Gohlis, »Giancarlo De Cataldo: Alles eine Erfindung?«, in: Die Zeit 2012/45, 11. Nov. URL: http://www.zeit.de/2012/45/Giancarlo-De-Cataldo-Zeit-der-Wut/ [10.02.2015]. 
Mit der Zeit hat diese Gruppe legendenhaften Charakter angenommen. Noch heute eignen sich einfache Kleinkriminelle ungeniert nicht existierende Verbindungen zur Bande an. Und eifrige Bullen erhöhen das Prestige einer bedeutungslosen Verhaftung, indem sie den diensthabenden Dieb mit der mittlerweile legendären Bande in Verbindung bringen. ${ }^{12}$

Die spätere Fiktionalisierung dieser Fallgeschichte bricht die monomedialen Grenzen auf und steht mittlerweile in einem Geflecht unterschiedlicher Verarbeitungen desselben Inhalts und Reaktionen darauf:

So wird erst durch De Cataldos Roman das Wirken der Banda della Magliana und die Verbindungen zwischen organisierter Kriminalität und Politik innerhalb und v.a. auch außerhalb Italiens einer breiteren Öffentlichkeit bekannt; es kommt somit zu einer »Popularisierung « eines juristischen Falles. ${ }^{13}$

Gleichzeitig wird der Text Vorlage für eine ganze Reihe intermedialer Weiterverarbeitungen wie den gleichnamigen Film von 2005 unter der Regie von Michele Placido, die 2-staffelige TV Serie unter der Regie von Stefano Sollima (2008, 2010), das Videospiel Romanzo criminale. The Game, zahlreiche Parodien (wie Manzo Criminale (dt.: das kriminelle Rind)) sowie ein von HBO geplantes amerikanisches Serien-Remake. Es kommt also zu einer Art multi- und transmedialer Kettenreaktion.

Die allgemeine Popularität des Falles (die durch die fast fluthafte transmediale Präsenz der Banda verstärkt wird) bewirkt dann wiederum zweierlei: zum einen werden die der Fiktion zugrundeliegenden Fakten beinahe zur Gänze von ihrer Faktualität befreit und nur mehr rein im Universum der Fiktion referentialisiert (wie ein regelrechter Hype v.a. um die Figuren der Serie, angeregte Diskussionen in Internetforen zu diversen Cliffhangern etc. beweisen). Zum anderen aber wird die Fiktion in die Realität rückgeführt, wenn es zu Imitationen und Identifikationen von

12 De Cataldo, (Anm. 1), 71. Orig.: »Con l'andar del tempo, la dimensione di questo gruppo avrebbe assunto contorni di leggenda. Ancora oggi piccoli delinquentelli $[\ldots]$ si appropriano con disinvoltura di quarti di nobiltà criminale millantando legami inesistenti con la Banda. E zelanti sbirri accrescono il prestigio di arresti periferici collegando arbitrariamente il ladro di turno alla ormai mitica Banda.«

13 Vgl. auch Donati, »Romanzo criminale: le pericolose interferenze tra realtà e finzione «, in: Milanesi (Hg.), Il romanzo poliziesco, la storia, la memoria, (2009), 447, (458). 
realen Kriminellen mit den Verbrechern aus Buch/Film/Serie kommt. So wird von einer Polizeioperation unter dem Namen »Romanzo criminale « berichtet, die am 8. Mai 2011 in Gallarate stattgefunden hat. ${ }^{14}$ Dabei handelt es sich um das Vorgehen gegen eine TeenagerDrogenhändlergruppe, die sich die Spitznamen der Protagonisten aus Romanzo criminale gegeben haben.

Und $\mathrm{zu}$ guter Letzt bewirkt De Cataldos fiktionaler Bestseller dokumentarische Gegen-Erzählungen, wie z.B. die 2004 erschienene dokumentarische Antwort des Staatsanwaltes Otello Lupacchini, einer der ersten, der gegen die Bande zu ermitteln begann: Banda della Magliana. Alleanza tra mafiosi, terroristi, spioni, politici, prelati... ${ }^{15}$

All diese Auswirkungen und Weiterverarbeitungen bedingen, dass Romanzo criminale als Gesamtphänomen betrachtet werden muss, da »[t]he multiplicity of fragments which compose Romanzo criminale corresponds to many photos of the same place taken from different perspectives: different genres, a variety of focalizations, alternative readings in diverging contexts. ${ }^{16}$ Marta Boni schlägt im Weiteren vor, Romanzo criminale, und darunter sind all dessen unterschiedliche mediale Versionen zu subsumieren, als »transmediales Narrativ«, d.h. als »transmediales Großprojekt« zu betrachten. Romanzo criminale gleiche demnach einem großen Puzzle, bei dem sich der Rezipient auf eine $»$ treasure hunt ${ }^{17}$ begibt. Henry Jenkins, der den Begriff des »transmedia storytelling « propagiert, versteht darunter folgendes:

Transmedia storytelling represents a process where integral elements of a fiction get dispersed systematically across multiple delivery channels for the purpose of creating a unified and coordinated entertainment experience. Ideally, each medium makes it[s] own unique contribution to the unfolding of the story. ${ }^{18}$

Selbst wenn der Begriff »transmedial « es in diesem Fall nicht punktgenau trifft, da das »Projekt Romanzo criminale« nicht von Anfang an als

14 Vgl. dazu die Zeitungsnotiz vom 9.3.2011, URL: http://www3.varesenews.it/comuni/gallarate/articolo.php?id=197938 [10.02.2015].

15 Dt.: Banda della Magliana. Eine Allianz aus Mafiosi, Terroristen, Spionen, Politikern, Prälaten....

16 Boni, Romanzo criminale: Transmedia and Beyond, 2013, 113.

17 Boni, (Anm. 16), 114.

18 Vgl. URL: http://henryjenkins.org/2007/03/transmedia_storytelling_101.html [10.02.2015]. 
transmediales Projekt intendiert war (also nicht »systematically « und mit einem bestimmten $»$ purpose $«),{ }^{19}$ sondern sich vielmehr verselbständigt hat, und die Transposition in andere Medien natürlich auch unter Gesichtspunkten der Vermarktung betrachtet werden muss, ist er dennoch aussagekräftig. Der Begriff ist insofern fruchtbar, als er deutlich macht, dass Romanzo criminale eben in seiner Gesamtheit zu betrachten ist, um dessen Auswirkungen auf die Wahrnehmung eines historischen Rechtskapitels erfassen zu können.

Dass eine populärwissenschaftliche Nacherzählung realer Rechtsfälle eine Wirkung über diese selbst hinaushaben kann, geht schon aus den vielzitierten Worten aus Schillers Titel zu seiner Pitaval-Sammlung (1792) hervor, nämlich: »Merkwürdige Rechtsfälle als ein Beitrag zur Geschichte der Menschheit «. ${ }^{20}$ Im Rahmen der Formel »Recht populär « ist nun die Frage zu stellen, welche Wirkung die Erzählung De Cataldos und ihre diversen Transpositionen auf die gesellschaftliche Verarbeitung und Wahrnehmung dieses Falles haben und welche besondere Rolle die Stellung des Autors als Richter und Autor spielt. Oder allgemeiner, bzw. an Goodman angelehnt, formuliert: Wie verhält sich diese »Vielheit wirklicher Welten ${ }^{21}{ }^{21}$ zueinander? Und in unserem konkreten Fall: Wie verhält sich die Welt der realen Rechtsgeschichte zu jener fiktiven, denn, so Goodman: »[...] die Künste als Modi der Entdeckung, Erschaffung und Erweiterung des Wissen [müssen] ebenso ernst genommen werden [...] wie die Wissenschaften $[\ldots] \ll^{22}$

19 Dass das Buch allerdings eine potentielle mediale Weiterverarbeitung schon vorweg beabsichtigt, vermutet ein Leser, wenn er in seiner Rezension schreibt: »Manchmal gewinnt man als Leser den Eindruck, als habe der Autor die Geschichte mit Absicht so strukturiert, dass sie leicht verfilmbar ist. Man könnte das Buch vielleicht sogar als Konzeptbuch bezeichnen. Falls De Cataldo wirklich diese Absicht hatte, dann war er mit einer Kino-Verfilmung und einer TV-Serie, mit seinem Buch als Grundlage, mehr als erfolgreich.« Tietz, »Der Leser als Beobachter. Eine Buchbesprechung $\mathrm{zu}>$ Romanzo criminale «, URL: »http://www.literaturcafe.de/buchkritiken-politthriller-romanzo-criminale/ [10.02.2015].

20 Der volle Titel lautet: Merkwürdige Rechtsfälle als ein Beitrag zur Geschichte der Menschheit. Nach dem französischen Werk des Pitaval durch mehrere Verfasser ausgearbeitet und mit einer Vorrede begleitet, herausgegeben von Schiller, Jena 1792.

21 Goodman, Weisen der Welterzeugung, 1990, 14.

22 Goodman, (Anm. 21), 127. 


\section{Romanzo criminale als faktuale Fiktion}

Romanzo criminale, sowohl der Text als auch der gleichnamige Film und die Serie, ist eine Art Banden-Biographie und zeigt detailreich den Werdegang von ein paar unorganisierten Ganoven zu einer genau konturierten Gruppe professionell agierender Krimineller, bis hin zu deren $»$ Untergang «, der teils durch Verrat innerhalb der Bande und darauffolgende Racheakte, teils durch den pentismo, d.h. das Kollaborieren einiger Mitglieder mit der Justiz, eingeleitet wird. Dem Genre des Thrillers gemä $\beta$ - und Romanzo criminale ist in der Tat eher dem Thriller- als dem Krimigenre zuzurechnen - gibt es neben einem fast durchwegs korrupten Polizeiapparat eine kleine Gruppe, die über ein Mindestmaß an Berufsmoral und -ethik verfügt und sich dem scheinbar sinnlosen Kampf der Überführung der Verbrecher widmet. Dabei kommen sie selbst in Gefahr und/oder werden ihrer beruflichen Integrität beraubt. Neben diesem klassischen Gut-Böse-Antagonismus gibt es allerdings noch eine dritte Instanz, »Il Vecchio«, der über der schier unerschütterlich scheinenden Macht und der Brutalität der Bande und jenseits der legalen Möglichkeiten der Justiz als eine Art »Übervater « steht. Diese namenlose und gottähnliche Figur ${ }^{23}$ hat letztendlich die Geschicke aller in der Hand. Wer gemeint ist, wird nicht näher definiert; es dürfte sich dabei aber wohl um die von Sandro Pertini - dem ehemaligen italienischen Staatspräsidenten - geprägte Bezeichnung für eine Art »Zentrale« handeln, die sowohl den rechten als auch linken Terrorismus leitet und aus Geheimdienstmitgliedern und Politikern (des In- und Auslandes) besteht. ${ }^{24}$ Wer Leiter dieser Zentrale gewesen sein könnte, sind reine Mutmaßungen. Mit dem »Il Vecchio « können aber historische Figuren wie Licio Gelli (Großmeister von P2), Federico Umberto D'Amato (Geheimdienstchef) ${ }^{25}$ und Giulio Andreotti (mehrmaliger Ministerpräsident und Vorsitzender der DC) in Verbindung gebracht werden. Definitiv handelt es sich um eine

23 In der deutschen Ausgabe bleibt Il Vecchio unübersetzt und wird mit »Vecchio « wiedergeben, was m.E. zu dem falschen Eindruck führt, dass es sich dabei doch um eine Art Namen handeln könnte.

24 Vgl. Locher, Bleierne Jahre. Linksterrorismus in medialen Aushandlungsprozessen in Italien 1970-1982, 2013, 274.

25 Diesen Namen nennt auch De Cataldo in einem Interview als einen der Drahtzieher, aber bestimmt nicht den einzigen; vgl. Antonello/O'Leary, »Sotto il segno della metafora: Una conversazione con Giancarlo De Cataldo«, in: the italianist 29 (2009), 350, (355). 
allegorische Figur für eine durch und durch korrumpierte und amoralische politische Gruppe.

Auf den ersten Blick ist die Faktenbasiertheit der Inhalte kaum evident. Dass es sich um die Darstellung einer der größten real existierenden Verbrecherbanden und ihrer juristischen Bekämpfung in der jüngeren italienischen Geschichte handelt, wird nur vereinzelt markiert. So gibt es im Roman kaum eindeutige dokumentarische Hinweise, keine Fußnoten, wenige übergenaue Hetero-Referentialisierungen (wie es z.B. den Konventionen des historischen Romans und dem dokumentarischen Modus entsprechen würde). Ähnliches gilt auch für den Film und für die Serie. Es gibt nur ein paar Schriftinserts mit Orts- und Zeitangaben. Filmtechnisch fügen sich aber beide in die Tradition des Mafiafilms und sind mit intermedialen Verweisen auf die ganz »Großen« des Genres von Francis Ford Coppolas Godfather bis zu Francesco Rosis Lucky Luciano gespickt. Eine »unkonventionelle« Filmtechnik ist somit weniger einem versucht dokumentarischen Modus als vielmehr dem Zitieren eines bestimmten Filmgenres zu verdanken.

Einzig der zeitliche Rahmen ist klar eingegrenzt. Die Handlung umfasst die Ereignisse zwischen 1977 und 1992. Die einzelnen Kapitelüberschriften bestehen jeweils aus der Jahreszahl und einem Stichwort die Handlung betreffend, z.B.: »Februar 1978. Abmachungen $\ll .{ }^{26}$ Den einzelnen Serienepisoden wird ebenfalls immer wieder eine Jahreszahl vorangestellt oder sie werden mit anderen (indirekten) Fremdreferenzen versehen. Wollen wir den fiktiven und faktualen Gehalt der Inhalte bestimmen, scheinen auf den ersten Blick die fiktiven zu dominieren, d.h. die Prämisse der Selbstreferentialität, wie sie in semantischen Fiktionalitätstheorien proklamiert wird, scheint zuzutreffen. Auch wenn Selbstreferentialität meist in Fiktionstheorien Eingang findet, die sich auf schriftliche Texte konzentrieren, kann diese hier auch auf die Frage der Referentialität der bildlichen Medien umgelegt werden. Dorrit Cohn bestimmt Fiktion als »nonreferential narrative «, als $»[\ldots]$ a work of fiction [that] creates [itself] the world to which it refers by referring to it. ${ }^{27}$ Demnach operiert der fiktionale Text primär mit Selbstreferenzen, der faktuale primär mit Fremdreferenzen. Romanzo criminale baut nun zu einem Großteil auf Selbstreferenzen auf, verfügt aber freilich auch über außerfiktional referentialisierbare Inhalte. Selbst-

26 De Cataldo, Romanzo criminale. Politthriller, aus dem Ital. von Karin Fleischanderl, 2010, 31.

27 Cohn, (Anm. 7), 13. 
und Fremdreferenzen scheinen dabei aber in einer Art Ping-Pong-Spiel beständig ihre Plätze zu tauschen und sind daher nicht eindeutig auf Fakt oder Fiktion festlegbar. Des Weiteren ist mitzubedenken, dass Referentialisierungen, von Rezipientenseite, nicht nur auf eine außerästhetische Wirklichkeit vorzunehmen versucht werden, sondern auch innerhalb der unterschiedlichen Medien, d.h. transmedial zwischen Buch, Film und Serie.

Diese Mischung aus Selbst-, Hetero- und Transreferentialität wird besonders in der Figurenkonstruktion deutlich. Prinzipiell gibt es eine Mischung aus neu, d.h. rein fiktional konstruierten Figuren (die auch Teil der Bande werden) und historischen Personen. Von den historisch referentialisierbaren Figuren wiederum werden einige mit neuen Namen versehen und bekommen, wie die realen Bandenmitglieder, auch jeweils (sprechende) Spitznamen: so entspricht der fiktive Bandenchef Pietro Proietti, besser bekannt unter seinem Spitznamen »Il Libanese «, dem realen Bandenchef Franco Giuseppucci. Einige wiederum behalten in der Fiktion den realen Spitznamen (so bekommt die fiktive Entsprechung Fluvio Luciolos, nämlich Maurizio Anibaldi, denselben Spitznamen, nämlich »Il Sorcio«, wie sein reales Gegenbild). Und wieder andere bekommen neue Spitznamen, allerdings innerhalb desselben semantischen Feldes. So trug der reale Massimo Carminati den Spitznamen »Il nazista «, woraus in der fiktionalen Welt der assoziative Name »Il nero« wird (der Schwarze $=$ der Faschist). Gerade diese (undurchsichtige) Mischung zwischen einer Totalfiktionalisierung, einer Quasifiktionalisierung und Hetero-Referentialität macht den besonderen Status des Textes aus.

Besonders deutlich wird der (intendierte) Hybridcharakter aber auf paratextueller Ebene mit dem Titel: »Romanzo criminale $\ll{ }^{28}$ Die explizite Betitelung als »romanzo« markiert selbstverständlich die unser Fiktionsbewusstsein wesentlich mitbestimmende ästhetische Differenz: Was folgt, ist ein Roman, und damit etwas Erfundenes, d.h. Nicht-Echtes; die Inhalte müssen demnach auch nur innerhalb der Welt dieses Romans referentialisierbar sein. Dass die Angabe »romanzo« allerdings nicht als Titelzusatz steht (wie so oft), sondern der Titel selbst ist, schlägt in Wahrheit eine paradoxe Rezeptionshaltung vor: »Du liest einen Roman, der ein Roman ist«, d.h. die Fiktionalität wird geradezu potenziert. Die Irreführung ist komplett, wenn man das Adjektiv »criminale« dazu denkt.

28 Der italienische Titel bleibt sowohl in den jeweiligen fremdsprachigen Übersetzungen (einzig im Spanischen wurde daraus »Roma criminal «), als auch in den anderen medialen Adaptionen (Serie, Film) erhalten. 
Zum einen könnte »romanzo criminale « ganz einfach als »Kriminalroman« übersetzt werden; Krimi heißt allerdings auf Italienisch giallo oder romanzo poliziesco. Die Komposition »romanzo criminale « ist daher eine an das Englische crime novel angelehnte (und idiomatisch nicht korrekte) Parallelübersetzung, eine Art »Lehnübersetzung«. Zum anderen kann es aber auch als Attribut gelesen werden, was dann soviel heißen würde wie »krimineller Roman«, d.h. ein Roman, der Kriminelles tut, weil er etwas erzählt, was er nicht erzählen sollte.

Mit Romanzo criminale haben wir es also mit einer Fiktion zu tun, die mit den Worten Elena Espositos - »zum Spiegel [wird], in dem die Gesellschaft ihre eigene Kontingenz reflektiert, die Normalität einer nicht mehr eindeutig festgelegten und bestimmbaren Welt $\ll .{ }^{29}$ Sie fährt fort, in Bezug auf fiction als Roman: »Indem der Roman eine realistische Fiktion anbietet, erlaubt er es den Beobachtern, Erwartungen und Analysen zu entwickeln, die die undurchsichtige reale Realität nicht zulassen würde. ${ }^{30}$ Der fiktionale Modus scheint also ein Ausweg zu sein, einer nicht entschlüsselbaren, nur fragmenthaft vorhandenen und widersprüchlichen Faktenlage Herr zu werden.

Die Hybridität dieses Gesamtwerks zwischen Fakt, Fiktion und Populärkultur soll im Zentrum der weiteren Ausführungen stehen. In einem ersten Schritt wird daher der Roman als faktuale Fiktion genauer betrachtet, da er zum einen den Ursprung der »transmedialen Kettenreaktion « bildet und zum anderen am stärksten eine Zwischenstellung zwischen »erfundener Geschichte«, »tatsächlicher Historie« und »nacherzählter Geschichte« einnimmt. Dabei sollen zwei Aspekte besondere Beachtung finden: die Autorrolle und das ambivalente Verhältnis von (erfahrener) Geschichte und (erzählter) Geschichte. In einem letzten Schritt soll das Werk auf transmedialer Ebene betrachtet und der dadurch entstehende Hybridcharakter analysiert werden.

\section{Hybridität I: die Autorrolle}

Romanzo criminale stellt unterschiedliche Autorrollen zur Diskussion und macht damit die Autorfigur auf mehreren Ebenen überdurchschnittlich stark präsent.

Zuallererst ist die besondere Rolle des realen Autors zu hinterfragen. Die Tatsache, dass es sich beim Autor um einen praktizierenden Juristen 
handelt, der obendrein in die reale Hintergrundgeschichte der Fiktion persönlich involviert war, lenkt auf paratextueller Ebene die Rezeptionshaltung insofern, als Heteroreferentialisierungen von Rezipientenseite geradezu angeregt werden. Besonders in deutschsprachigen Medien, in denen über die deutsche Übersetzung des Buches berichtet wurde, wird die Richterrolle des Autors hervorgehoben; ${ }^{31}$ in den italienischen Medien scheint das etwas weniger der Fall zu sein. Fakt ist aber, dass - v.a. auch in der Sekundärliteratur ungewöhnlich oft auf Interviews mit dem Autor rekurriert wird, um so die besondere Stellung des Textes zwischen bekannten historischen Tatsachen, erfundenen Inhalten und geschichtlichen Hypothesen zu diskutieren. De Cataldo selbst distanziert sich in diesen Interviews immer wieder, sowohl von dem investigativen Charakter seines Romans als auch von angeblichen Erleichterungen, die ihm die Expertise aufgrund seines Richterberufs beim Schreiben solch eines »historischen Thrillers« bringe:

»Glauben Sie, dass Profis, seien es Richter, Staatsanwälte oder Polizisten, die Krimis schreiben, einen Vorteil gegenüber jenen Krimiautoren haben, die von Beruf nur Schriftsteller sind « - »Absolut nicht. Mittlerweile steht die technische Information (wie man ein Protokoll schreibt bis zum Beschreiben einer Autopsie), die das Produkt eines Laien von dem eines Profis unterscheiden würde, jedem zur Verfügung. $\ll^{32}$

Dass, selbst wenn der Autor das gerne hätte, es in diesem Fall nicht gelingt, den realen Autor von seinem Text zu trennen, liegt eben am Charakter des Textes einer faktualen Fiktion, denn gerade »faktuale Erzählungen räumen ihrem Urheber [...] einen prominenten Platz ein, um ihren Wahrheitsgehalt durch Zeugen- und Zeitgenossenschaft $\mathrm{zu}$ verbürgen. $\ll^{33}$

31 Vgl. z.B. den Bericht in Die Zeit: Gohlis, (Anm. 11).

32 Interview, (Anm 2); Orig.: »Pensa che i professionisti, quali magistrati e poliziotti, che scrivono gialli abbiano qualcosa in più dei giallisti che fanno solo gli scrittori?< - >Assolutamente no. Ormai il livello di informazione tecnica (da come si redige un verbale a come si descrive un'autopsia) che distingue un prodotto ingenuo da uno professionale è alla portata di tutti.«. Vgl. auch: Sørensen, »Letteratura noir e storiografia. Le voci del doppio Stato in Romanzo criminale di De Cataldo«, in: Jansen/Khamal (Hg.), Memoria in Noir. Un'indagine pluridisciplinare, 2010, 87, (96).

33 Koschorke, Wahrheit und Erfindung. Grundzüge einer Allgemeinen Erzähltheorie, 2012, 88. 
Genau diese Urheberrolle wird über die Erzählinstanz gespiegelt. Der Roman ist durch eine durchwegs (fast unglaubwürdig) auktoriale Erzählperspektive gekennzeichnet. Diese Art gottähnlicher Erzähler scheint (mit einem leicht ironischen Unterton) den (idealen) Richterblick bzw. das omnipräsente Auge des Gesetzes zu imitieren - das in unserem Fall zwar alles sieht, aber doch kein Recht walten lassen kann. Die Erzählung selbst wird immer wieder durch pseudo-faktuale oder dokumentarische Inserts unterbrochen: unkommentiert zwischen den narrativen Kapiteln »hängen« Gerichtsakten, Vernehmungsprotokolle, Polizeinotizen, Zeitungsartikel und Gesetzeszitate.

»Verkompliziert « wird diese scheinbar »reine« Erzählperspektive dann durch zwei Alter Ego-Figuren des Autors. Dazu zählt einmal »Il Vecchio«, der - wie erwähnt - auf histoire-Ebene die Fäden in der Hand hält und als Allmacht dargestellt wird und damit als paradoxale Alter EgoFigur des Autors fungiert. Ist er zum einen klar »negativ« gezeichnet, d.h. geht die Sympathielenkung nicht zu seinen Gunsten aus, ist er zum anderen in seiner Klar- und Weitsicht wiederum ein Spiegel eines Erzählers bzw. Urhebers, der meint, die Geschichte durchschaut zu haben:

Vecchio verspürte eine tiefe Verachtung für die sogenannten Großen dieser Welt. Bankiers, Unternehmer, Politiker und gekrönte Häupter, die meinten, sie würden die Fäden in der Hand halten, waren für ihn nur ein Haufen dummer, mittelmäßiger Hasardeure. Leute die nicht fähig waren, die Handlung in ihrer Tragweite zu verstehen. $^{34}$

Zum anderen wird aber auch der (auf der »guten Seite « stehende) Commissario Scialoja als autorähnlicher Urheber präsentiert: In einem fiktiven Interview für den Corriere Romano zeigt sich Kommissar Scialoja ebenfalls als eine Art Alter Ego des realen Autors. An einem Punkt höchster Resignation angelangt, beschreibt er ohne Zurückhaltung die Bande und ihr Wirken und vor allem ihre Vernetzungen bis in höchste Staatskreise folgendermaßen:

34 De Cataldo, (Anm. 26, 2010), 195f. Orig.: »Il Vecchio provava un profondo disprezzo per i cosiddetti grandi della Terra. Considerava banchieri, trafficanti, politici e teste coronate che si illudevano di reggere le fila del gioco una manica di stolti, mediocri avventurieri. Gente incapace di percepire la trama nel suo complesso.«De Cataldo, Romanzo criminale, 2002, 216. (meine Hervorhebung). 
»Wir sind beinahe ins Herz des korrupten Systems vorgedrungen. Beinahe. Wir sind zufällig dorthin gelangt, bei den Ermittlungen zu der Ermordung eines unbedeutenden Kriminellen. Wir haben unglaubliche Dinge in Erfahrung gebracht. Die Spur führt von der »römischen Mafia«, wie ich sie nenne, über die Ermordung Moros und das Massaker von Bologna über Morde, die in den letzten zehn Jahren begangen wurden, direkt in den Bunker einer Verbrecherhorde im Dienste des Staates. Zu einer Sektion, die es offiziell gar nicht gibt, mit dem Phantom eines Chefs, bei dem die Fäden der größten Geheimnisse der jüngsten Geschichte zusammenlaufen. Und wir waren drauf und dran, diese Geschichte neu zu schreiben. Dann ... dann hat jemand einen Rückzieher gemacht. Namen sind unbedeutend. Man hat uns zu verstehen gegeben, dass eine gewisse Grenze nicht überschritten werden darf. Jemand hat die Botschaft empfangen und die Konsequenzen gezogen. Und wir stehen wieder am Anfang. Vielleicht ist dieses Land reich, wie Sie sagen. Aber es ist durch und durch korrupt, glauben Sie mir! « »Die Geschichte neu schreiben! Was für ein ehrgeiziges Ziel! Aber glauben Sie [...], dass es wirklich Aufgabe eines Richters oder eines Polizisten ist, die Geschichte neu zu schreiben?«

$»$ Solange es niemand sonst macht $\ldots \ll \ll^{35}$

Nicht nur wird hier in einer den Inhalt betreffenden mise en abyme de l'enoncé der Gesamttext gespiegelt, sondern auch die reale Erzählinstanz sowie die Erzählintention offen gelegt: Es geht darum, aus einem sozialen Gewissen heraus, die »Geschichte neu zu schreiben« und das, weil »es sonst niemand macht $\ll$.

35 De Cataldo, (Anm. 26, 2010), 495f. Orig.: »)Eravamo un passo dal cuore putrido dell'affare. Un passo, uno solo. C'eravamo arrivati per caso, indagando sull'omicidio di un malavitoso di mezza tacca. Abbiamo scoperto cose incredibili. Un filo che partiva da quella che io chiamo >la mafia romana e passava per l'uccisione di Moro, la strage di Bologna, dieci anni di omicidi, e portava nel bunker di una branca speciale direttamente dipendente dagli apparati dello Stato. Una sezione che ufficialmente non esiste, con un capo fantasma che è il crocevia di tutti i piú grandi misteri della Storia recente. E noi quella Storia stavamo per riscriverla. Poi...poi qualcuno si è tirato indietro. I nomi non hanno importanza. Ci hanno fatto capire che oltre un certo limite non ci sarebbe stato possibile spingerci. Quel qualcuno ha raccolto il messaggio e si è comportato di conseguenza. E ora siamo punto e daccapo. Questo Paese sarà anche ricco, come dice lei. Ma è marcio dentro, mi creda $<$ ! $>$ Riscrivere la Storia! Che traguardo ambizioso! Ma non crede che il proposito di riscrivere la Storia esuli dai compiti di un magistrato o di un poliziotto ? >Visto che non lo fa nessun altro... « Cataldo, (Anm. 34, 2002), 540f. 
Neben einem externen realen Richterautor und einem beinah übertrieben allwissenden Erzähler, werden also in diversen mises en abymes fiktive Autortypen zur Diskussion gestellt, die konträrer nicht sein könnten: Während der eine den Durchblick und die Macht hat, hat der andere zwar (offensichtlich) auch den Durchblick, aber keine Mittel, mit seinem Wissen etwas anzufangen. Indem diese Allmacht der einen Seite gegen die Ohnmacht der anderen ausgespielt wird, wird eben eine positive Vereinbarkeit von Wissen, Macht und Moral negiert.

In einer ironischen Schlusspointe wählt am Ende »Il Vecchio« just den Kommissar Scialoja zu seinem Nachfolger aus:

Vor zehn Monaten war Vecchio infolge des dritten Infarkts gestorben. Einige Zeit später hatte Scialoja ein Paket ohne Absender bekommen. Es enthielt Vecchios Tagebücher. Auf den beigelegten Kärtchen stand: »Viel Vergnügen beim Spielen». [...] Er besaß Vecchios Tagebücher! Er war der Hüter der geheimen Geschichte der Republik! [...] Gewiss, man würde aufräumen, aber mit Vernunft. [...] Er würde Vecchios Linie beibehalten. Im Schatten bleiben. In einem Büro am Rand, im Schutz eines neutralen Etikettes, mit einer Truppe von Haudegen, die bereit waren, beim kleinsten Wink loszupreschen. Ach, das Spiel, das Spiel! Sie alle in der Hand haben, als anonymer, gleichgültiger Schiedsrichter ihrer Schicksale! ${ }^{36}$

Dass diese Rollenübernahme gleichzeitig auch eine Niederlage ist, bestätigt der allerletzte Satz des Romans: »Aus welchen dunklen Ecken der Vergangenheit drang im Augenblick des Triumphes dieses unvergleichliche Gefühl der Niederlage hervor «? $?^{37}$

\section{Hybridität II: Geschichte vs. Geschichte}

36 De Cataldo, (Anm. 26, 2010), 569f. Orig.: »Dieci mesi prima il terzo infarto aveva stroncato il Vecchio. Qualche tempo dopo Scialoja aveva ricevuto un pacco anonimo. Conteneva i diari del Vecchio. Il biglietto d'accompagnamento diceva >Buon gioco! [ [...] Lui aveva i diari del Vecchio! Era il depositario della storia segreta della Repubblica! [...] Aveva il potere. Era il potere. [...] Si sarebbe fatta pulizia, certo, ma non juicio. [...] Avrebbe seguito la linea del Vecchio. Restare nell'ombra. In un ufficio periferico, protetto da una sigla anodina, con un manipolo di tagliagole pronti a scattare al minimo battito di ciglia. Ah, il gioco, il gioco! Stringerli tutti tra le dita, essere l'anonimo, indifferente arbitro dei loro destini! « De Cataldo, (Anm. 34, 2002), $624 \mathrm{f}$.

37 De Cataldo, (Anm. 26, 2010), 560. Orig.: »Nel momento del trionfo, da quali mai oscuri recessi del passato affiorava questo incomparabile senso di sconfitta $\ll$ ? De Cataldo, (Anm. 34, 2002), 625. 
Der »carattere di dramma-documentario del romanzo « ${ }^{38}$ wie es Sørensen nennt, ist eines der meistdiskutierten Themen in Bezug auf Romanzo criminale. Die Handlung ist eine Mischung aus Fakt und Fiktion, d.h. eine Mischung aus Real-Geschichte, neu erfundener und alternativer Geschichte. Der Autor De Cataldo selbst wird allerdings nicht müde, zu betonen, dass es sich um eine rein fiktive Erzählung handelt, die keinen Anspruch auf Wahrheit und Vollständigkeit legt. Vielmehr sei ihm daran gelegen, den Mythos der Banda zu bearbeiten:

Der Grundfehler liegt darin, Romanzo criminale als eine Geschichte der Banda della Magliana zu betrachten. Noch bevor sie von den Gerichtsakten und -urteilen geschrieben wurde, wurde die wahre Geschichte dieser kriminellen Holding von Bianconi, Flamini und anderen bereits hervorragend niedergeschrieben. Die Aufgabe des Erzählers ist es, die Geschichte zu hintergehen (die schön wäre - so Tolstoi - wenn sie nur wahr wäre), indem er sie den Bedürfnissen des Mythos anpasst. Aus den nackten Tatsachen eine metaphorische und mythologische Linie finden und auf das Herz einer falschen Geschichte hinweisen: daher ist sie oft wahrer, zumindest überzeugender, als die »offizielle « Version. ${ }^{39}$

Gleichzeitig macht er sich aber auch für das Argument der »Metaphernhaftigkeit « seiner Geschichte stark, wenn er in diversen Interviews mit einem, zugegebenermaßen, etwas fragwürdigen und unreflektierten Metaphernbegriff von der »storia come metafora spricht. ${ }^{40} \mathrm{Zu}$ meinen scheint er, dass jedem geschichtlichen Ereignis eine Art universell gültiger Mechanismus innewohnt, der als solcher auch auf andere geschichtliche Ereignisse übertragbar ist. In dem, dem Roman Nelle mani giuste (Dt.: »Schmutzige Hände«) - die chronologische

38 Sørensen, (Anm. 32), 101.

39 D'Attis, »Intervista a Giancarlo de Cataldo«, URL: http://www.blackmailmag.com/Intervista_a_Giancarlo_De_Cataldo.html [29.1.2015]. Orig.: $» L$ 'errore di fondo sta nel considerare Romanzo Criminale come una storia della Banda della Magliana. Prima ancora che dai rapporti giudiziari e dalle sentenze, la vera storia di questa holding criminale è stata ottimamente scritta da Bianconi, Flamini e altri. Il compito del narratore è di tradire la storia (che sarebbe bella cosa, diceva Tolstoi, se solo fosse vera) piegandola alle esigenze del Mito. Estrarre dai nudi fatti una linea metaforica e mitologica e puntare al cuore di una falsa storia: per ciò stesso più vera, e comunque più convincente, di quella >ufficiale «."

40 Vgl. Antonello/O'Leary, (Anm. 25), 357f.; vgl. auch Amici, «Dall'epopea criminale all'ambiguità dei giorni nostri«, in: Jansen/Khamal, (Anm. 32), 77, (78). 
Fortsetzung von Romanzo criminale - vorangestellten »Hinweis für den Leser « legt der Erzähler zwar den direkten Bezug zu einer konkreten historischen Epoche offen, distanziert sich davon aber alsbald im Nebensatz, indem er die Ereignisse einer bestimmten Zeit (nämlich der 1980er und 90er Jahre in Italien) als beispielhaft auch für andere dieser Art bestimmt. De Cataldo präzisiert diesen Vorgang im Weiteren, wenn er meint:

Abgesehen von den bewusst genannten realen Personen sind die Protagonisten dieses Romans jedoch frei erfunden; Firmennamen, öffentliche Strukturen, Medien und Politiker werden nur genannt, um Figuren, Bilder und das Wesen der kollektiven Träume zu benennen, die sie wesentlich mitgeprägt haben. Nur die metaphorische Überhöhung gestattet es, die Personen, die dem Autor als Vorbild gedient haben, in literarische Archetypen zu verwandeln. ${ }^{41}$

Schließlich nimmt er noch konkret auf eine von ihm vorgestellte Neuinterpretation eines konkreten historischen Ereignisses Bezug (»Was die Autobombe im römischen Olympiastadion betrifft, entwirft der Roman eine These, die dem Ergebnis des Florentiner Prozesses widerspricht $[\ldots] . \ll^{42}$ ), um dann mit einem von ihm in unterschiedlichen Kontexten gern bemühten indirekten Zitat Tolstois abzuschließen: »Im Übrigen halte ich es mit Tolstoi, der meinte, die Geschichte wäre eine schöne Sache, wenn sie nur wahr wäre. Dieses hier aber ist schließlich nur ein Roman «. ${ }^{43}$

Gerade die Notwendigkeit des realen wie auch impliziten Autors (in Nelle mani giuste), die Überzeitlichkeit der Ereignisse zu betonen, bringt das »Dilemma « zwischen Geschichte (im Sinne von Historie) und Geschichte (im Sinne von Erzählung) zum Ausdruck. In diesem

41 De Cataldo, Schmutzige Hände. Politthriller, aus dem Ital. von Karin Fleischanderl, 2011, 5. Orig.: »Tuttavia, a eccezione delle personalità espressamente citate, i personaggi di questo romanzo sono frutto di fantasia e i nomi di aziende, strutture istituzionali, media e personaggi politici vengono utilizzati soltanto al fine di denotare figure, immagini e sostanze dei sogni collettivi che sono stati formulati intorno a essi. È la Metafora a trasformare in archetipi letterari le persone che possono aver fornito spunti di ispirazione all'autore «. De Cataldo, Nelle mani giuste, 2007, 2.

42 De Cataldo, (Anm. 41, 2011), 5. Orig.: »Circa l'autobomba allo stadio Olimpico, l'interpretazione fornita dal libro si discosta da quanto asserito nel processo di Firenze $\ll$ De Cataldo (Anm. 41, 2007), 2.

43 De Cataldo, (Anm. 41, 2011), 6. Orig.: »D'altronde, ho sempre pensato, con Tolstoj, che la Storia sarebbe una cosa bella, se solo fosse vera. E questo, in definitiva, è solo un romanzo« De Cataldo (Anm. 41, 2007), 2. 
Homonym (Geschichte vs. Geschichte) sieht der Historiker Koselleck nicht nur eine Übereinstimmung auf lautlicher und Schriftebene, sondern auch eine »semantische Konvergenz $«,{ }^{44}$ denn die Frage, die sich stelle, sei nicht die nach Faktualität auf Seiten der Historiker und Fiktionalität auf Seiten der Schriftsteller/Künstler, vielmehr gehe es um das Verhältnis zwischen »nieder-geschriebener Geschichte « und »erfahrener Geschichte«: »Wie verhält sich die sprachliche Konstitution einer Geschichte, sei es die Geschichte eines historischen, eines poetischen oder sonst eines Schriftstellers, zu dem, was nunmehr als geschichtliche Wirklichkeit erfahren und bezeichnet wird? ${ }^{45}$ Es scheint also eine »unaufhebbare Spannung, die es verhindert, daß irgendeine Sprachhandlung jemals geschichtliche Wirklichkeit einholen kann ${ }^{46}{ }^{46} \mathrm{zu}$ bestehen, denn Geschichte ist immer ein bereits Vergangenes. Damit stehen Schriftsteller (und auch der von Koselleck als »historisierende Schriftsteller ${ }^{47}$ bezeichnete) vor demselben Problem wie Historiker, nämlich vor der »Inkommensurabilität von geschichtlicher Wirklichkeit und ihrer sprachlichen Verarbeitung ${ }^{48}$ (sie reagieren lediglich darauf auf unterschiedliche Art und Weise). Die Wiedergabe einer vergangenen Wirklichkeit ist damit immer die »Fiktion des Faktischen «. ${ }^{49}$ Ebendies deckt sich mit dem von De Cataldo so gerne bemühten Tolstoi-Zitat, nämlich: »die Geschichte wäre eine schöne Sache, wenn sie nur wahr wäre«.

\section{Hybridität III: Entreferentialisierung durch transmediale Verschiebung: die Serie}

Die Komplexität von Romanzo criminale liegt aber nicht nur in dem Hybridcharakter zwischen erzählter und Realgeschichte, sondern auch in seinem Charakter eines transmedia narrative. So stellt sich eben nicht nur die Frage nach Selbst- und Heteroreferenzen, sondern auch die nach »Transreferenzen«. Die einzelnen Teile des transmedialen Gesamtwerkes hängen lose zusammen, unterscheiden sich teilweise auch, ergänzen sich aber vor allem. Daher müssen sie, neben den notwendigen Unterschieden,

44 Koselleck, »Fiktion und geschichtliche Wirklichkeit«, in: Zeitschrift für Ideengeschichte. »Die Rückkehr der Wahrheit «2007/1.3, 39, 44.

45 Koselleck, (Anm. 44), 44f.

46 Koselleck, (Anm. 44), 47.

47 Koselleck, (Anm. 44), 52.

48 Koselleck, (Anm. 44), 48.

49 Koselleck, (Anm. 44), 50. 
v.a. über einen gewissen Grad an Wiedererkennungselementen verfügen. In unserem Fall sind diese zahlreich. Neben dem inhaltlichen Grundschema (das wenig verändert in allen Medien wieder auftaucht, auch deshalb, weil in allen Fällen der Autor De Cataldo wesentlich mitbeteiligt war - als Buch- bzw. als Drehbuchautor für Film und Serie) wurde v.a. auf eine peinlich genaue Figurenkonstruktion geachtet: So wurden die Darsteller der Serie aufgrund ihrer physiognomischen und physischen Ähnlichkeit mit den Schauspielern des Films ausgewählt und diese wiederum anhand ihrer Ähnlichkeit mit den historischen Figuren. ${ }^{50}$

Das Aufspüren dieser Transreferenzen, d.h. der direkten oder indirekten Bezugnahmen auf andere mediale Verarbeitungen desselben Stoffes, gleicht einer, schon weiter oben erwähnten, »Schatzsuche«: »A transmedial world can be thought of as a streasure hunt $<$. The principles of >spreadability< and >drillability< refer to a consumer's enjoyment when searching for details, analyzing and comparing sources. $«{ }^{51}$ Marta Boni selbst untersucht in ihrer umfassenden Studie eben genau dieses »Konsumverhalten «, indem sie Diskussionen in Online-Foren und Chats analysiert, in denen, v.a. im Zusammenhang mit der Serie, über Handlungsverläufe diskutiert wird, die sich von jenen des Films und Buchs unterscheiden, aber auch immer wieder über möglicherweise andere »Ausgänge « spekuliert wird. So zeigt Boni, dass z.B. der Mord am 1. Bandenchef »Il Libanese « (Franco Giuseppucci), der in der Serie als Cliffhanger am Ende der 1. Staffel inszeniert wird, heftige Diskussionen auslöst, in denen spekuliert wird, dass er vielleicht doch nicht gestorben sein möge oder wer der Mörder sein könnte. ${ }^{52}$ Gerade der Tod des Libanesen ist aber eine den meisten Rezipienten sowohl aus der Geschichte als auch aus dem Buch und Film wohlbekannte Tatsache.

Es ist v.a. die Serie, die solche von mir als »Transreferentialisierungen « bezeichneten und letztlich eben auch Entreferentialisierungen auslöst. International gesehen ist die Serie das erfolgreichste Produkt der Marke Romanzo criminale. Neben einer begeisterten Aufnahme durch das italienische Publikum feierte sie einen enormen Erfolg in Frankreich und wurde auch im deutschsprachigen Raum viel gesehen. Der Hype um die

50 Zur Veranschaulichung vgl. das Bildmaterial in: »I volti criminali diventano $>$ di serie $<$ la critica«, URL: https://nottecriminale.wordpress.com/2010/07/27/i-volticriminali-diventano-di-serie-la-critica/ [11.02.2015].

51 Boni, (Anm. 16), 114

52 Vgl. Boni, »Romanzo Criminale: la série dans un récit transmédial «, in: Mise au point 2011/3. URL: http://map.revues.org/957 [10.02.2015]. 
Serie, der sich auch in einer höchst erfolgreichen Fanartikel-Industrie zeigt (so gibt es z.B. T-Shirts mit den Konterfeis der kriminellen Helden), ist v.a. mit der gelungenen Genremischung aus Mafiafilm gespickt mit romantischen Elementen und Historiendrama zu erklären. Der Stoff selbst eignet sich besonders gut für das Serienformat, denn im Prinzip besteht die Handlung aus der variierten Wiederholung desselben. Die bestimmende repetitive Handlungsreihe ist - schematisch zusammengefasst -: Verbrechensidee und -planung, Ausführung, Probleme, Kampf, Mord/Tod/Gefängnis (und dies so lange, bis ein Großteil der Protagonisten tot, geflüchtet oder im Gefängnis ist). Neben dieser Basis-Handlungsreihe gibt es Szenen und Szenenabfolgen, die sich in fast gleicher Weise wiederholen. Eine davon wäre die Szene, in der ein Bandenmitglied vor dem Gefängnis auf ein anderes Mitglied wartet, um dieses abzuholen und dann in die Bar Franco zu bringen, in der sich die Bande üblicherweise trifft. Schauplatz, Perspektiven und Kameraeinstellung sind jedes Mal beinahe identisch. Gerade diese immer wiederkehrende Variation eines Grundschemas macht den Film ${ }^{53}$ und auch das Buch ${ }^{54}$ etwas schwerfällig, wohingegen eben die Serie dadurch den Spannungsbogen von Episode zu Episode aufrecht erhalten kann.

Zum Serienerfolg trägt auch die Tatsache bei, dass die Bandenmitglieder noch stärker als in Film und Buch in den Fokus gerückt und auch in ihrer Ambivalenz gezeigt werden. Die Erzählperspektive ist nun fast ausschließlich die Perspektive der Bandenmitglieder und sie lösen, trotz der sie charakterisierenden, beinahe monströsen Brutalität, immer wieder Sympathie aus. Dies gelingt wiederum durch das Einfügen "privater « Geschichten: in analeptischen Sprüngen werden die Vorgeschichten gezeigt und damit das jeweilige Verhalten erklärt und

53 Vgl. z.B. die BBC Rezension: „But the picture's ambition is thwarted by numbing repetition (you're never more than two scenes away from the next ruthless assassination) and a fatal lack of rooting interest in the characters, who don't have the charm to go with their cheekbones. To draw comparisons with a true gangster classic like The Godfather or GoodFellas would be simply Criminale «. Leyland,

»Romanzo Criminale«, URL: http://www.bbc.co.uk/films/2006/10/31/ romanzo criminale_2006_review.shtml [10.02.2015].

54 Vgl. z.B. die Rezension von Jürgen Priester, URL: http://www.krimicouch.de/krimis/giancarlo-de-cataldo-romanzo-criminale.html [10.02.2015]. Er beendet seine Rezension mit folgendem Fazit: »Es ist schwer, für Romanzo Criminale eine Empfehlung auszusprechen. Es zählt mit Sicherheit nicht zur leicht konsumierbaren Kriminalliteratur. Es ist sperrig und bedarf der Muße. Politisch Interessierte sollten auf jeden Fall einen Blick riskieren «. 
auch bis zu einem gewissen Grad gerechtfertigt. Daraus entsteht ein (paradox anmutendes) »Identifikationspotential« mit den Protagonisten, wie ein Online-Zuschauer-Kommentar beweist: »criminals who look human because of their closeness. Dressed like my parents when they were young, Romans like me, and who grew up and lived not far from me ${ }^{55}$ Es ist gerade der point of view des $»$ Bösen $«{ }^{56}$ der diese italienische Serie als untypisch italienisch gelten lässt und auch Anlass zu Polemisierungen gegeben hat. Aufgrund der allzu vielen und heftigen Gewaltszenen wurde die Serie stark kritisiert, da die Gewalt in dem Universum der banda ihre Berechtigung zu haben und die einzige logische Konsequenz menschlichen Handelns zu sein scheint. Der Bürgermeister von Rom hat daher in einer »Zufriedenheits-Befragung « (2009) der Bürger Roms u.a. die Frage gestellt, ob man der Meinung sei, dass die Serie Romanzo criminale Einfluss auf die aktuelle Jugendkriminalität in Rom haben könne: $45 \%$ der Befragten antworteten mit ja, 24\% mit nein, $31 \%$ enthielten sich. ${ }^{57}$ In der Folge gab es mehrere Boykott-Aufrufe. Betont werden muss allerdings, dass nur die erste Staffel den Blickwinkel des Bösen als positive Sichtweise suggeriert, die zweite Staffel zeigt den langsamen Untergang und die anfänglich stolzen Helden sind $\mathrm{zu}$ Gefangenen, Geflohenen oder von anderen Verbrechern Gekauften geworden. Also gilt am Ende doch: »Crime does not pay«.

Der Erfolg der Serie liegt aber v.a. auch in einer sehr gelungenen, und für europäische Serien untypischen, Filmtechnik. Die aufwendige mise en scène zeigt sich in einem überzeugenden Retro-Look; die vordergründige, übermarkierte Montage wird v.a. in den »künstlerisch « (beinahe "pasolinesk «) anmutenden Kampfszenen evident, die mit Parallelmontagen und jump cuts geschnitten sind und von einer meist »kontraintuitiven « Musik begleitet werden: Je stärker die Gewalt, desto »leichter « die Musik, scheint das Motto zu sein. Gerade dieses Element spielt auch Kritikern in die Hände, die der Serie vorwerfen, Gewalt zu verherrlichen.

Die Serie, und damit vorerst der Gipfel der transmedialen Weiterverarbeitung, bringt nun die Rezipienten am stärksten dazu,

55 Aus mymovie; zit. nach Boni, (Anm. 16), 99.

56 Sorrentino spricht von einem »punto di vista di Caino «. Sorrentino, »Due bandi criminali e il doppio stato: De Cataldo, Romanzo criminale e Manotti, Le corps noir«, in: Milanesi, (Anm. 13), 461, (462).

$57 \mathrm{Vgl}$.

http://www.comune.roma.it/wps/portal/pcr?contentId=NEW110042\&jp_pagecod e=newsview.wp\&ahew=contentId:jp_pagecode [10.02.2015]. 
Transreferentialisierungen vorzunehmen. Das bestätigt auch Boni, die meint: »For the novel and the movie, there wasn't the same interest in discussing the dangers [of] Romanzo criminale. The real controversy broke out in social networks of media from the airing of the series in $2008 \ll^{58}$ Gefragt wird weniger: wer und was entsprechen den realen Figuren und Ereignissen der Realgeschichte? Vielmehr interessiert zum einen: wer entspricht welcher Figur in Buch und Film, und zum anderen: wer ist das »coolste « Vorbild, wer hat am meisten Identifikationspotential? Die Verbrecherfiguren, um die ein Kult entsteht, werden somit ihres historischen Ursprungs entledigt, d.h. »entreferentialisiert«, gleichzeitig aber als fiktionale Figuren in die Realität rückgeführt, wie sich am Beispiel der Jugendbande zeigt, die sich mit Namen der fiktiven Verbrecher schmückt.

\section{Schlussfolgerung}

Das Besondere an Romanzo criminale ist nun zum einen, wie gezeigt wurde, dass es sich um die Fiktionalisierung eines realen Verbrechensund Rechtsfalles durch einen der realen Richter selbst handelt. Zum anderen bietet diese in der Folge die Vorlage für eine ganze Reihe an intermedialen Weiterverarbeitungen, d.h. also es scheint, dass erst durch dieses transmediale (fiktionale) Großwerk einer der größten Verbrechensund Rechtsfälle der jüngeren italienischen Geschichte Teil des kollektiven Gedächtnisses und der kulturellen Identität wird.

Albrecht Koschorke würde dies im Zuge seiner »Allgemeinen Erzähltheorie« bestätigen, wenn er meint:

In Gestalt von Narrativen kann sich ursprünglich frei Erfundenes im kollektiven Bewusstsein sedimentieren und $\mathrm{zu}$ einer harten sozialen Tatsache werden. [...] $[\mathrm{N}]$ arrative Elemente »vereigentlichen « sich, jedenfalls wird ihre Uneigentlichkeit während dieser Metamorphose vergessen. ${ }^{59}$

Gleichzeitig spricht er aber auch von einer reziproken Einflussnahme (wie wir sie auch im Fall von Romanzo criminale gesehen haben), nämlich:

Andererseits hat die Reflexion auf das Erzählen eine zutiefst lösende Kraft. Sie ist imstande, Tatsachen, die unumstößlich schienen, als Redeweisen von gestern zu 
entdinglichen. Auf diese Weise verwandelt sich »Eigentliches « in »Uneigentlichkeit«, »Wesenhaftes« in ein »Spiel der Zeichen« zurück. ${ }^{60}$

In eine ähnliche Kerbe schlägt Ansgar Nünning, der ebenso die Erzählforschung für die Kulturwissenschaften öffnen möchte und für eine »kulturwissenschaftliche Narratologie« plädiert. Nünning argumentiert mit der These von der »Narrativität von Kulturen ${ }^{61}$ und stützt seine Überlegungen auf das Prinzip der »Kulturen als Erzählgemeinschaften« (nach Müller-Funk). ${ }^{62}$ Sinngemäß begreift er Erzählungen als Modi der Weltkonstruktion (angelehnt an Goodmans Begriff des »ways of worldmaking «) und betont die performative Kraft des Erzählens. Dabei schlägt er ein (an Ricoeur angelehntes) dreistufiges Modell vor, das das Verhältnis zwischen Erzählungen bzw. kulturellen Narrativen auf der einen Seite und den Kontexten bzw. Kulturen auf der anderen Seite beschreibt: nämlich Präfiguration, Konfiguration und Refiguration. ${ }^{63}$ Demnach sind Erzählungen zuerst durch die außerliterarische Wirklichkeit präformiert (Präfiguration), diese Wirklichkeit wird durch unterschiedliche Darstellungsmodi zum Ausdruck gebracht (Konfiguration) und schließlich wirkt diese narrative Inszenierung von Welten auf die außertextuelle Wirklichkeit zurück (Refiguration). Am Beispiel von Romanzo criminale sind diese drei Stufen unschwer zu erkennen. Auf der Ebene der Präfiguration haben wir die reale Bande und ihre Geschichte der 1970er und 1980er Jahre; diese wiederum wird fiktional in Buch, Film und Serie verarbeitet (=Konfiguration). Und gerade aufgrund dieser Fiktionalisierungen kommt es in der Folge zu weiteren Texten (wie eben die Sachbücher zur Bande etc.) und zu neuen, an die fiktionale Bande angelehnten, Verbrechervereinigungen (=Refiguration). Diese Refiguration bestätigt auch Marta Boni, die zu dem Schluss kommt: »Romanzo criminale ist $\mathrm{zu}$ einem gesellschaftlichen Phänomen geworden. Die Figuren, ihre Dialoge und die übersättigten

60 Koschorke, (Anm. 33), 24.

61 Nünning, »Wie Erzählungen Kulturen erzeugen: Prämissen, Konzepte und Perspektiven für eine kulturwissenschaftliche Narratologie«, in: Strohmaier (Hg.), Kultur - Wissen - Narration. Perspektiven transdisziplinärer Erzählforschung für die Kulturwissenschaften, 2013, 15, (27).

62 Vgl. Nünning, (Anm. 61), 15f. und 28.

63 Nünning, (Anm. 61), 32. 
Farben der Inszenierung sind mittlerweile zu einer Art Code geworden, der von einem breiten Publikum geteilt wird. ${ }^{64}$

Im Falle von Romanzo criminale könnte man also von einem kulturellen emplotment (qua Hayden White) sprechen, nämlich genauer »ein[em] faktuale[n] emplotment, [das] narrativen Lückenschluss des Realen [betreibt] $\ll .{ }^{65}$ De Cataldo scheint es damit gelungen zu sein, durch die Transposition eines realen Rechtsfalles in die fiktionale Welt, eine korrumpierte und gänzlich ohne Rechtsbewusstsein lebende MikroGesellschaft publik zu machen und aus dem Eck der Historiographie herauszuholen und so einem größeren Publikum zur Diskussion zu stellen.

64 Boni, (Anm. 52). Orig.: »Romanzo criminale est devenu un phénomène sociétal. Les personnages, leurs répliques et les couleurs saturées des décors sont désormais une sorte de code partagé par un vaste public.«

65 Tiller, (Anm. 3), 41 